\title{
Effects of external field wavelength and solvation on the Photophysical Property and Optical Nonlinearity of 1,3-Thiazolium-5-Thiolates Mesoionic Compound
}

\author{
Shugui Hua, ${ }^{\dagger}$ Zeyu Liu, ${ }^{* \star}$ and Tian Lu, ${ }^{* \S}$ \\ †College of Life Science and Chemistry, Jiangsu Key Laboratory of Biological Functional \\ Molecules, Jiangsu Second Normal University, Nanjing 210013, People 's Republic of China \\ ${ }^{\ddagger}$ School of Environmental and Chemical Engineering, Jiangsu University of Science and \\ Technology, Zhenjiang 212018, People's Republic of China
}

${ }^{\S}$ Beijing Kein Research Center for Natural Sciences, Beijing 100022, People’s Republic of China

*Email: liuzy@just.edu.cn.

*Email: sobereva@sina.com. 


\begin{abstract}
The photophysical property and optical nonlinearity of an electronic push-pull mesoionic compond, 2-(4-trifluoromethophenyl)-3-methyl-4-(4-methoxyphenyl)-1,3thiazole-5-thiolate were theoretically investigated with a reliable computing strategy. The essence of the optical properties were then explored through a variety of wave function analysis methods, such as the natural transition orbital analysis, hole-electron analysis, (hyper)polarizability density analysis, decomposition of the (hyper)polarizability contribution by numerical integration, and (hyper)polarizability tensor analysis, at the level of electronic structures. The influence of the electric field and solvation on the electron absorption spectra and (hyper)polarizabilities of the molecule are highlighted and clarified. This work will help people to understand the influence of external field wavelength and solvent on the optical properties of mesoionic-based molecules, and provide a theoretical reference for the rational design of chromophores with adjustable properties in the future.
\end{abstract}

\title{
Graphical abstract
}

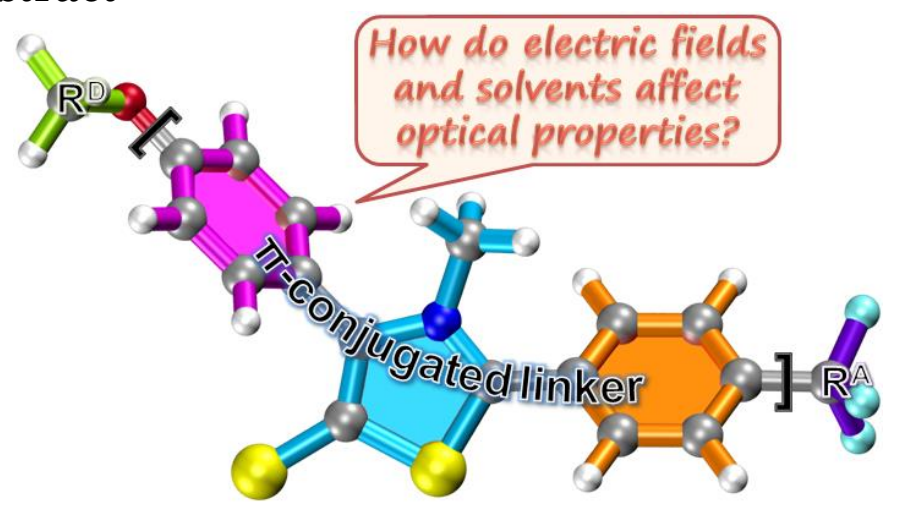




\section{Introduction}

Organic optoelectronic materials, which can be adopted in various optical devices such as photoelectric sensors, optical information processing and storage devices, and optical communication elements, have always been one of the hot spots of people's attention and research. ${ }^{1-3}$ The excellent optical response characteristics of material molecules are the premise for them to be used to construct advanced photoelectric functional materials. Therefore, the field of optical materials has been exploring how to develop new compounds with tunable photophysical properties or enhanced (hyper)polarizabilities for a long time. ${ }^{4-6}$

So far, researchers have successfully recognized a variety of molecules with exciting optical properties, including inorganic complexes, ${ }^{7-9}$ organic molecules, ${ }^{10-13}$ metal clusters, ${ }^{14,15}$ doped polarizable electrides, ${ }^{16,17}$ and even macrocyclic compounds with special topology, ${ }^{6,18,19}$ and applied some of them into practice. Among them, the conjugated organic chromophores with electron donating and electron accepting groups at both ends were one of the most popular optical units, partially due to their convenient structural tailoring. Generally speaking, the photophysical and nonlinear optical (NLO) properties of these chromophores can be controlled by adjusting their structural modules: electron donor, $\pi$-conjugated linker, or electron acceptor. ${ }^{20-24}$

Since Baker and coworkers put forward the concept of "mesoionic" in 1949,,$^{25,26}$ mesoionic rings defined by planar five- or six-membered heterocyclic betaines with at least one side-chain whose $\alpha$-atom in the ring plane have been recognized as a promising candidate for conjugated bridges of optical materials because of their electronic mobility. ${ }^{27}$ Numerous mesoionic compounds with different electron donors and electron acceptors have been studied both theoretically and experimentally. ${ }^{28-31}$ Recently, for example, Barbosa-Silva et al. prepared three mesoionic compounds and measured the optical properties of them by Hyper-Rayleigh scattering (HRS) experiment. ${ }^{32}$ Our theoretical calculation for the same systems not only perfectly reproduced the electronic absorption spectra and first-order hyperpolarizabilities observed in experiment, but more importantly, it deeply revealed the essences of the optical properties of these mesoionic molecules from the electronic structure level. ${ }^{33}$ At the same time, we also found that Lyra and collaborators had theoretically predicted the first-order static hyperpolarizability on a variety of mesoionic compounds including the above-mentioned three ones by employing the semi- 
empirical time-dependent Hartree-Fock (AM1-TDHF) method. ${ }^{34}$ However, due to the constraints of the level of calculation adopted, the response properties of these molecules obtained by them are obviously different from the measurements of the experiment and those calculated by us with a more reliable strategy. ${ }^{32,33}$

In view of the fact that the influence of external field wavelength and solvent usually used to adjust the optical properties of organic materials has not been reported yet for mesoionic systems, in this work, we selects an electronic push-pull mesoionic compound, 2-(4-trifluoromethophenyl)-3-methyl-4-(4-methoxyphenyl)-1,3-thiazole5-thiolate (hereinafter referred to as MIC), that possessing substituents with strong electron-donating/accepting capacity and also involving in previous calculation studies $^{34}$ as the research object to explore the influence of environments on the optical properties of this kind of organic molecules.

\section{Computational Details}

The structure of the MIC was optimized at the PBE0 $0^{35} /$ def-TZVP ${ }^{36}$ level in dimethylsulfoxide $\left(\mathrm{C}_{2} \mathrm{H}_{6} \mathrm{OS}\right)$ solution, which is the actual environment of the similar experimental studies. ${ }^{32}$ The optimized geometry is characterized to be stable point on potential energy surface with no imaginary frequency. Then, the electron absorption spectrum and (hyper)polarizability of the molecule were calculated in vacuum and four solvents, including dichloromethane $\left(\mathrm{CH}_{2} \mathrm{Cl}_{2}, \varepsilon=8.93\right)$, acetone $\left[\left(\mathrm{CH}_{2}\right)_{2} \mathrm{CO}, \varepsilon=\right.$ 20.49], acetonitrile $\left(\mathrm{C}_{2} \mathrm{H}_{3} \mathrm{~N}, \varepsilon=35.69\right)$, and $\mathrm{C}_{2} \mathrm{H}_{6} \mathrm{OS}(\varepsilon=46.83)$. The solvation model based on density (SMD) was adopted to consider solvation effects on molecular structure and properties. ${ }^{37}$ The excitation energy and oscillator strength of the lowlying singlet states of the MIC were studied with the time-dependent density functional theory (TD-DFT) ${ }^{38,39}$ at the PBE0/def2-TZVP ${ }^{40}$ level. The molecular (hyper)polarizability and (hyper)polarizability density were evaluated using analytic derivatives of the system energy (namely, coupled-perturbed Kohn-Sham method, $\mathrm{CPKS})^{41}$ and finite difference of electron density, respectively, at the CAMB3LYP ${ }^{42} /$ aug-cc-pVTZ(-f,-d) level, where aug-cc-pVTZ(-f,-d) is a reduced version of the aug-cc-Pvtz ${ }^{43}$ basis set with the removal of $f$-type polarization functions of nonhydrogen atoms and $d$-type polarization functions of hydrogen atoms. The selections of PBE0 functional in calculating the excited state and CAM-B3LYP functional in evaluating molecular nonlinearity have been proved to be reasonable by many 
theoretical works..$^{33,44-46}$ The decompositions of the (hyper)polarizability components into each structural unit were obtained by numerical integrations of the (hyper)polarizability density in the space of every atom partitioned by Becke's method. ${ }^{47}$ For the formulas of the (hyper)polarizability, (hyper)polarizability density, and (hyper)polarizability decompositions, see the descriptions in the Supporting Information.

All (TD-)DFT calculations were performed with the Gaussian 16 program. $\underline{48}$ The wave function analyses were implemented in Multiwfn 3.7 code, ${ }^{49,50}$ and the visualizations of the isosurface maps were realized with VMD software. ${ }^{51}$

\section{Results and Discussion}

The Cartesian coordinates of the ground-state MIC in $\mathrm{C}_{2} \mathrm{H}_{6} \mathrm{OS}$ solution are available in Table S1, and the molecular structure is illustrated in Scheme 1.

Scheme 1. Structure of the MIC studied in this work ${ }^{a}$

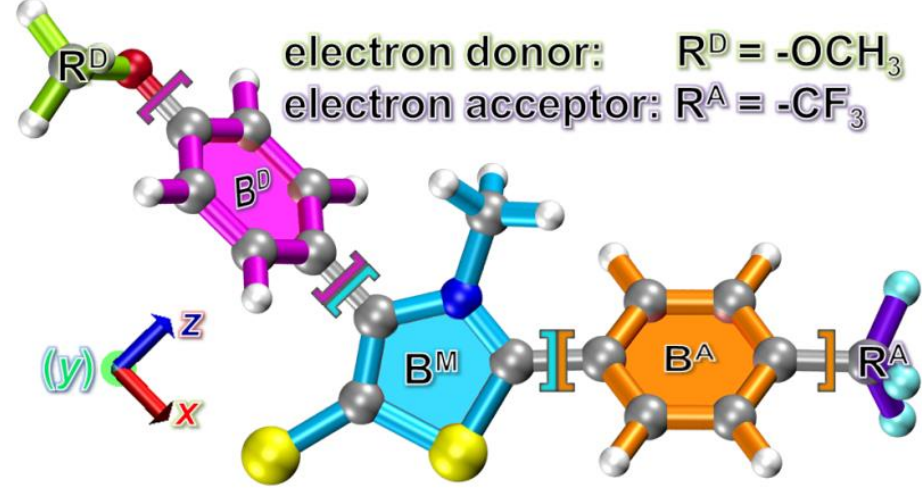

${ }^{a}$ Also shown is the Cartesian axis. Atom color code: white, H; grey, C; blue, N; red, O; cyan, F; yellow, S.

\subsection{Electron Absorption Spectrum and the Nature of Electron Transition}

The selected data related to electron transitions for the MIC are listed in Table S2. The calculated absorption band of the molecule in vacuum almost covers the whole visible region of $420-800 \mathrm{~nm}$, as shown in Figure 1. The strongest absorption is located at $548 \mathrm{~nm}$ followed by a peak at ultraviolet range of $333 \mathrm{~nm}$ with medium intensity. As also can be seen from the figure, solvation effect makes the two absorption bands shift significantly towards the short-wave direction regardless of the polarity of the solvent, accompanied by the enhancement and attenuation in the 
absorption intensity of the maximum absorption peak and its companion, respectively. However, there is no remarkable distinction of the absorption spectra in different solvents, and specifically speaking, with the increase of the solvent polarity, the absorption peak of the MIC shows a slight blue-shift from $481 \mathrm{~nm}$ in $\mathrm{CH}_{2} \mathrm{Cl}_{2}$ and seems to converge to a fixed value of $466 \mathrm{~nm}$, but the absorption intensity of the spectrum does not show any regular change.

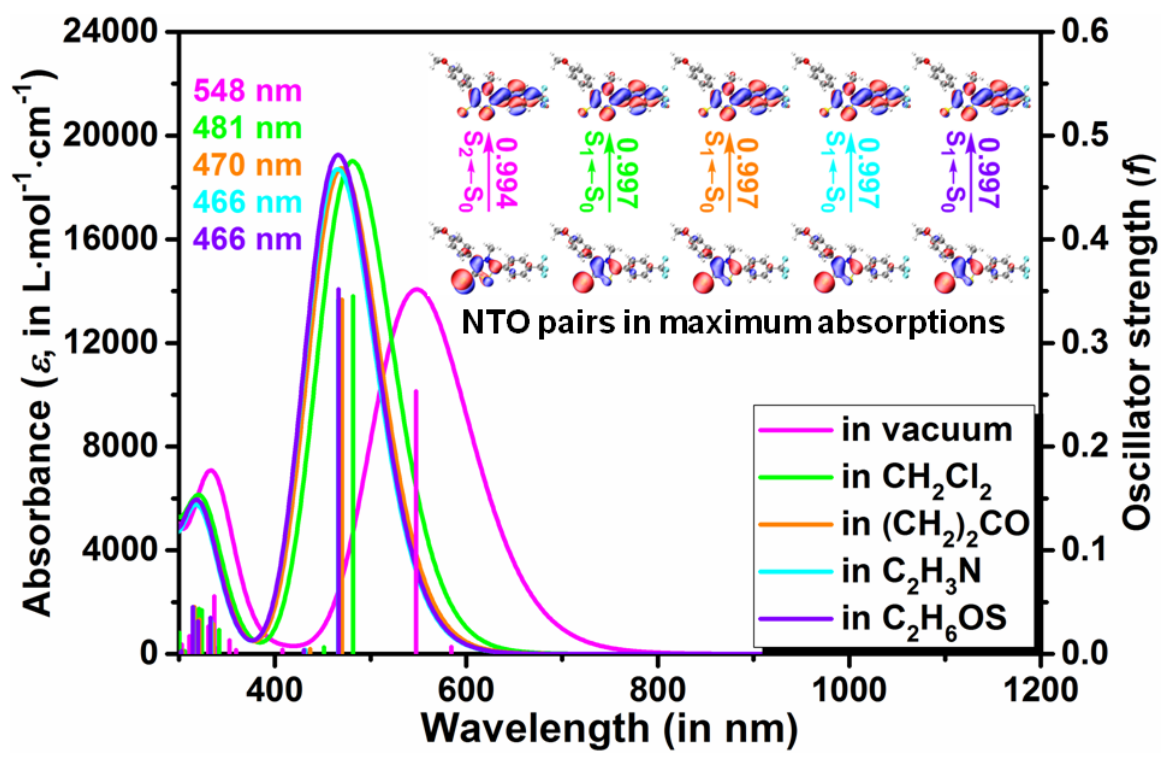

Figure 1. Electron absorption spectra for the MIC. A Gaussian function has been employed for broadening with a full width at half-maximum of $2000 \mathrm{~cm}^{-1}$. The inset shows dominant natural transition orbital pairs (isovalue $=0.03 \mathrm{au}$ ) involved in maximum absorptions, blue and red regions denote positive and negative orbital phases, respectively. The labelings on the inset denote the critical excited states associated with electron transitions and the corresponding eigenvalues of natural transition orbital pairs.

For each maximum absorption, we performed the natural transition orbital (NTO $)^{52}$ analysis to visualize the orbital characteristics in the process of electron transition. The inset in Figure 1 shows the isosurfaces of critical NTO hole/particle pairs of the MIC in various environments. It can be seen that the NTO pairs (mainly in the NTO hole) of the molecule in vacuum are only slightly different from those in solvents, while those in various solvents are indistinguishable. Therefore, it is not difficult to understand why the MIC displays very similar UV-Vis absorption spectra in solvents while subtly different one in vacuum. Further analysis shows that all maximum 
absorptions of the MIC are attributed to the electron transitions from lone-pair orbital, $n$, of $\mathrm{S}$ atoms and $\pi$-bonding orbital at mesoionic ring $\left(\mathrm{B}^{\mathrm{M}}\right)$ to $\pi^{*}$-antibonding orbital distributed at the $\mathrm{B}^{\mathrm{M}}$ and benzene unit $\left(\mathrm{B}^{\mathrm{A}}\right)$ connected to the electron acceptor $\left(\mathrm{R}^{\mathrm{A}}=-\right.$ $\mathrm{CF}_{3}$ ), namely, $n \rightarrow \pi^{*}$ and $\pi \rightarrow \pi^{*}$ electron excitations on the molecular skeleton, and the former is more significant.

Figure S1 shows the highest occupied molecular orbitals (HOMOs) and the lowest unoccupied molecular orbitals (LUMOs) of the MIC in various environments. A comparison between Figures 1 and S1 shows that the distribution of NTO hole/particle related to the maximum absorption of the MIC is very similar to its HOMO/LUMO, which suggests that the HOMO and LUMO are the crucial orbitals during the maximum absorption. Indeed, for the electron excitation in the maximum absorption process, the calculated contribution of HOMO-LUMO transition in vacuum is more than $92 \%$, and the proportion of this transition in various solvents is nearly $99 \%$. The blue-shift of the maximum absorption peak caused by solvation can therefore be reasonably explained by the fact that the HOMO-LUMO gap difference of MIC in solutions is significantly larger than that in vacuum, as shown in Figure S1. Oscillator strength $(f)$ of an excited state is proportional to the square of transition dipole moment $\left(\mu^{\mathrm{T}}\right)$ corresponding to the state. ${ }^{53}$ From the $\mu^{\mathrm{T}}$ of the molecule during the maximum absorption listed in Table S2, one can see that their changing trends in different environments are completely consistent with those of corresponding $f$ value.

The hole-electron analysis ${ }^{53}$ can give a more definite picture about electron excitation, which simply means that the regions where excited electron leaves and arrives are represented by hole and electron respectively and they are integrated into the same visual image. It can be inferred from Figure 2 that the main excitation characteristic of the studied molecule is that the hole and electron are concentrated on the mesoionic ring $\left(\mathrm{B}^{\mathrm{M}}\right)$ and the benzene ring $\left(\mathrm{B}^{\mathrm{A}}\right)$ attached to the electron-accepting group $\left(\mathrm{R}^{\mathrm{A}}=-\mathrm{CF}_{3}\right)$, respectively, and both $n$ and $\pi$ molecular orbitals participate in the excitation, which is very consistent with NTO and HOMO/LUMO analyses.

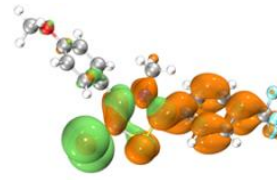

in vacuum $\left(S_{0} \rightarrow S_{2}\right)$

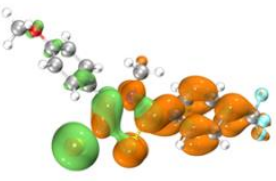

in $\mathrm{CH}_{2} \mathrm{Cl}_{2}$

$\left(S_{0} \rightarrow S_{1}\right)$

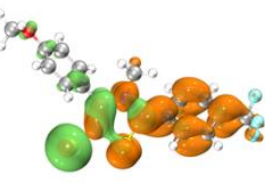

in $\left(\mathrm{CH}_{2}\right)_{2} \mathrm{CO}$

$\left(S_{0} \rightarrow S_{1}\right)$

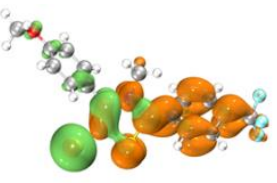

in $\mathrm{C}_{2} \mathrm{H}_{3} \mathrm{~N}$

$\left(\mathrm{S}_{0} \rightarrow \mathrm{S}_{1}\right)$

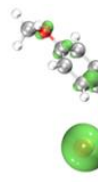

in $\mathrm{C}_{2} \mathrm{H}_{6} \mathrm{OS}$

$\left(S_{0} \rightarrow S_{1}\right)$ 
Figure 2. Real space representation of hole and electron distributions (isovalue $=$ 0.001) of the MIC. Green and orange regions denote the hole and electron distributions, respectively.

\subsection{Molecular (Hyper)Polarizability and Its Essential Feature}

As in our previous paper about mesoionic compounds, ${ }^{33}$ we found that when the fundamental wavelength of external field reaches a certain size, the molecular dipole moment and (hyper)polarizabilities will converge to a specific value. In solution, the convergence value is different from the calculated static one, but the two values are the same in vacuum. Considering the comparison in Ref. 33 between our calculated values and the corresponding experimental results, we believe that the convergence value at infinite wavelength rather than response value calculated under static conditions is a credible quantity that can be compared with the experimental measurement. Therefore, the response properties described below with "zerofrequency limit" is the calculated value at $99999 \mathrm{~nm}$ incident light, which is the maximum incident wavelength setting that Gaussian program accept in this kind of calculation. The molecular nonlinearity at other five different frequencies of incident light $(\lambda=1907,1460,1340,1180$, and $1064 \mathrm{~nm}$, respectively) are also calculated for comparison.

\subsubsection{HRS-Derived First-Order Hyperpolarizability and Dipolar/Octupolar}

\section{Contributions to the Second-Order NLO Activity}

In order to provide predictions for HRS experiment, we calculated the HRS-derived first-order hyperpolarizabilities $\beta_{\mathrm{HRS}}(-2 \omega ; \omega, \omega)$ [abbreviated $\beta_{\mathrm{HRS}}(\lambda)$, where $\lambda$ denotes the incident wavelength] of the MIC under six fundamental wavelengths of incident light, and summarizes important physics quantities in Table S3.

Our $\beta_{\mathrm{HRS}}(\lambda)$ value of MIC at the zero-frequency limit in vacuum is $27.9 \times 10^{-30} \mathrm{esu}$, which is smaller than the calculated result of $41.7 \times 10^{-30}$ esu by Lyra et al. ${ }^{34}$ This discrepancy is believed to be due to the relatively rough AM1-TDHF method used in their calculations. Similar situations have been observed in calculating the hyperpolarizabilities of other mesoionic compounds. ${ }^{33}$ The HRS-derived first-order hyperpolarizability of the MIC in this work enlarges with the increase of the external field frequency, that is, as shown in Figure $3(\mathrm{a})$, the order of $\beta_{\mathrm{HRS}}(\lambda)$ is: $\beta_{\mathrm{HRS}}(99999 \mathrm{~nm})<\beta_{\mathrm{HRS}}(1907 \mathrm{~nm})<\beta_{\mathrm{HRS}}(1460 \mathrm{~nm})<\beta_{\mathrm{HRS}}(1340 \mathrm{~nm})<\beta_{\mathrm{HRS}}(1180 \mathrm{~nm})<$ 
$\beta_{\mathrm{HRS}}(1064 \mathrm{~nm})$, indicating the obvious resonance effect of the external field on the molecular optical nonlinearity. In addition, we can see that the $\beta_{\mathrm{HRS}}(\lambda)$ in solvent is lower than that in vacuum at a specific frequency, which reveals that the solvation has an induced weakening effect on the response properties of the studied system, while the calculated values in different solvents, by contrast, are not much different.
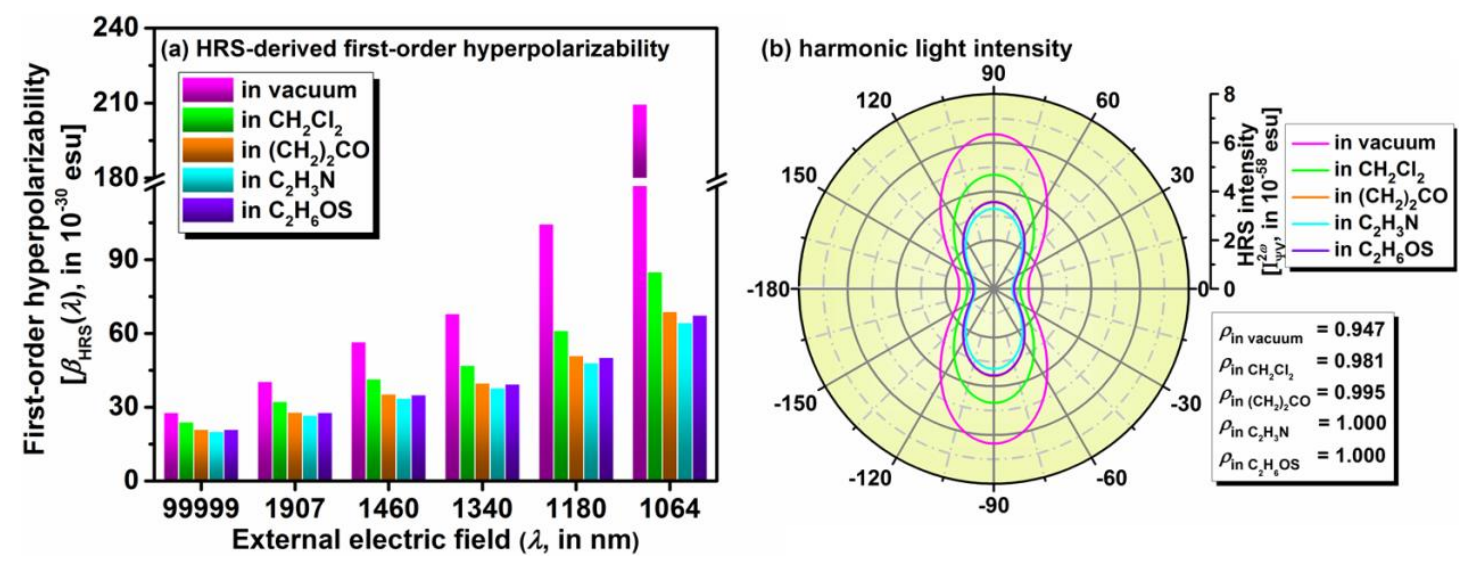

Figure 3. (a) Diagrams of HRS-derived first-order hyperpolarizabilities $\left[\beta_{\mathrm{HRS}}(\lambda)\right]$ of the MIC and (b) harmonic light intensity $\left(\mathrm{I}_{\Psi \mathrm{V}}^{2 \omega}\right)$ for the MIC as a function of the polarization angle $(\Psi)$ by polar representation at the zero-frequency limit, the inset shows the calculated nonlinear anisotropy parameters $(\rho)$ in different environments.

Polarization scanning of HRS intensity was performed to explore the contribution of dipolar/octupolar to the NLO activity of the MIC. As can be inferred from the nonlinear anisotropy parameter $(\rho)$ and depolarization ratio $(D R)$ in Table S3, the MIC dispalys the intermediate characteristic with almost equal dipolar and octupolar contributions at the zero-frequency limit in all environments. With the increase of the frequency of the external field, the dipolar contribution of the NLO activity enlarges gradually, making the MIC show more and more obvious dipolar features. On the other hand, the polar environment is conducive to balance the dipolar/octupolar contributions of the molecule, which promotes the MIC to show more obvious intermediate molecular characteristic in strong polar solvents. More detailed analysis from the diagram of normalized HRS intensity $\left(\mathrm{I}_{\Psi \mathrm{V}}^{2 \omega}\right)$ versus polarization angle $(\Psi)$ in Figures 3(b) and S2 shows that the $\mathrm{I}_{\Psi \mathrm{V}}^{2 \omega}$ of the molecule gradually decreases at any $\Psi$ with the increase of solvent polarity and significantly enhances with the increasing 
frequency of the external field especially for those in vacuum. These trends of molecular HRS intensity in different environments is completely consistent with those of HRS-derived first-order hyperpolarizability.

\subsubsection{EFISHG-Derived (Hyper)Polarizability and Its Essential Feature}

In general, the prerequisite for a bulk material to exhibit excellent NLO properties is that its constituent molecules have a large (hyper)polarizability. In order to estimate the potential application of the studied molecule in nonlinear optics, we calculated its (hyper)polarizabilities, $\alpha(-\omega ; \omega), \beta(-2 \omega ; \omega, \omega)$, and $\gamma(-2 \omega ; \omega, \omega, 0)$ [abbreviated $\alpha(\lambda), \beta(\lambda)$, and $\gamma(\lambda)$, respectively, where $\lambda$ denotes the incident wavelength], related to the electric-field-induced second-harmonic generation (EFISHG) technique. Then, the nonlinear essences of the optical response of the MIC were further investigated by the (hyper)polarizability density analysis, the decomposition of the (hyper)polarizability contribution by numerical integration, and the (hyper)polarizability tensor analysis.

Components of molecular (hyper)polarizability The selected components of (hyper)polarizabilities of the MIC at different external fields are arranged in Table S4. As can be seen, the $x$-component of the (hyper)polarizability accounts for a considerable proportion, and the contribution of the $z$-component to the (hyper)polarizability is not ignorable although it is small. By comparison, the $y$ component of molecular response properties, especially for the first-order and secondorder hyperpolarizabilities, is relatively trivial.

It is helpful to study the structural origin of the response property by decomposing the overall axial (hyper)polarizability component of molecules into the contributions of their constituent units, which can provide a reference for exploring how to modify and tailor the structure of organic molecules to improve their optical nonlinearity. The integrand functions of the (hyper)polarizability densities, that is, the local contributions of the (hyper)polarizability tensor, in each Cartesian axis $\left[-i \rho_{i}^{(1)}(\stackrel{r}{r})\right.$, $-i \rho_{i i}^{(2)}(\stackrel{\Gamma}{r})$, and $-i \rho_{i i i}^{(3)}(r), i=x, y$, and $\left.z\right]$ of the MIC under the zero-frequency limit in vacuum are displayed in Figure 4. The isosurfaces of the integrand functions accurately reflect the contribution of the (hyper)polarizability components of the MIC in each axis direction discussed above, that is, the $x$-component $>$ the $z$-component $>$ the $y$-component, and the positive and negative contributions of each region of the 
molecule to its (hyper)polarizability component are also clearly distinguished in the plot. It needs to be mentioned that the details of the contour surface of the integrand functions of the (hyper)polarizability densities are dependent on the molecular coordinates, while the corresponding (hyper)polarizability density functions in Figure S3 have no such limitation.

\section{(a) isosurface of $-i \rho_{i}^{(1)}(\vec{r})$ function}

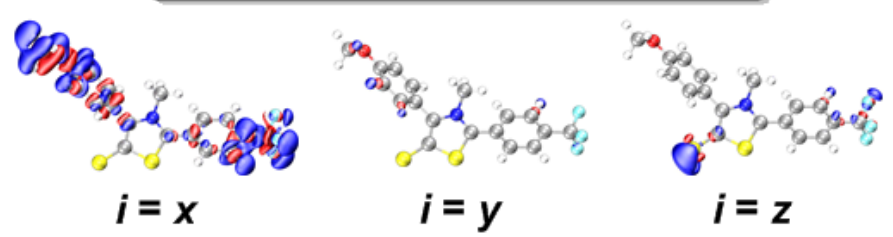

(b) isosurface of $-i \rho_{i i}^{(2)}(\vec{r})$ function

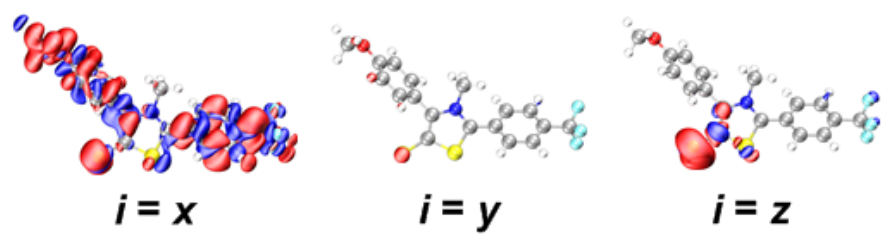

(c) isosurface of $-i \rho_{i i i}^{(3)}(\vec{r})$ function

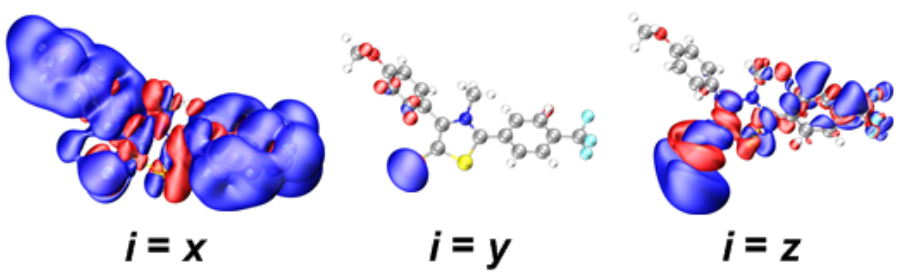

Figure 4. Plots of local contribution functions of (hyper)polarizabilities tensor of the MIC under the zero-frequency limit in vacuum: (a) $-i \rho_{i}^{(1)}(r)$ for the polarizability $($ isovalue $=1.0 \mathrm{au}),(\mathrm{b})-i \rho_{i i}^{(2)}(\stackrel{\mathrm{r}}{r})$ for the first-order hyperpolarizability (isovalue $=$ $10.0 \mathrm{au})$, and $(\mathrm{c})-i \rho_{i i i}^{(3)}(\stackrel{\mathrm{r}}{r})$ for the second-order hyperpolarizability (isovalue $=100.0$ au). Blue and red regions denote positive and negative contributions, respectively.

We also integrated the local contributions of the (hyper)polarizability tensor of each atomic region to quantify the contribution of molecular fragments to the (hyper)polarizability components. The decomposed (hyper)polarizability values of each structural unit are listed in Table S5 and plotted in Figure 5. By comparing Tables S5 and S4, we can see that the overall components of the (hyper)polarizability of the MIC estimated by numerical integration is very consistent with those calculated by the analytic derivative method, thus ensuring the reliability of our 
(hyper)polarizability decomposition research. Figure 5(a) shows that the components of molecular polarizability in all three Cartesian directions contribute to each structural unit in a certain amount, which eventually leads to the insignificant difference of polarizabilities in three coordinates. In contrast, it can be seen from Figure 5(b) and (c), except that the $\mathrm{B}^{\mathrm{M}}$ unit of the molecule contributes little to the second-order hyperpolarizability components in all directions, the hyperpolarizability component of the MIC in the $x$-axis far exceeds that in the $y$-and $z$-directions. In contrast, the hyperpolarizability component has a little share in the $z$-axis, but it is almost zero in the $y$-axis. This leads to the dominance of $x$-axis in the contribution of molecular hyperpolarizability and $y$-axis also accounts for a small part, as discussed above.

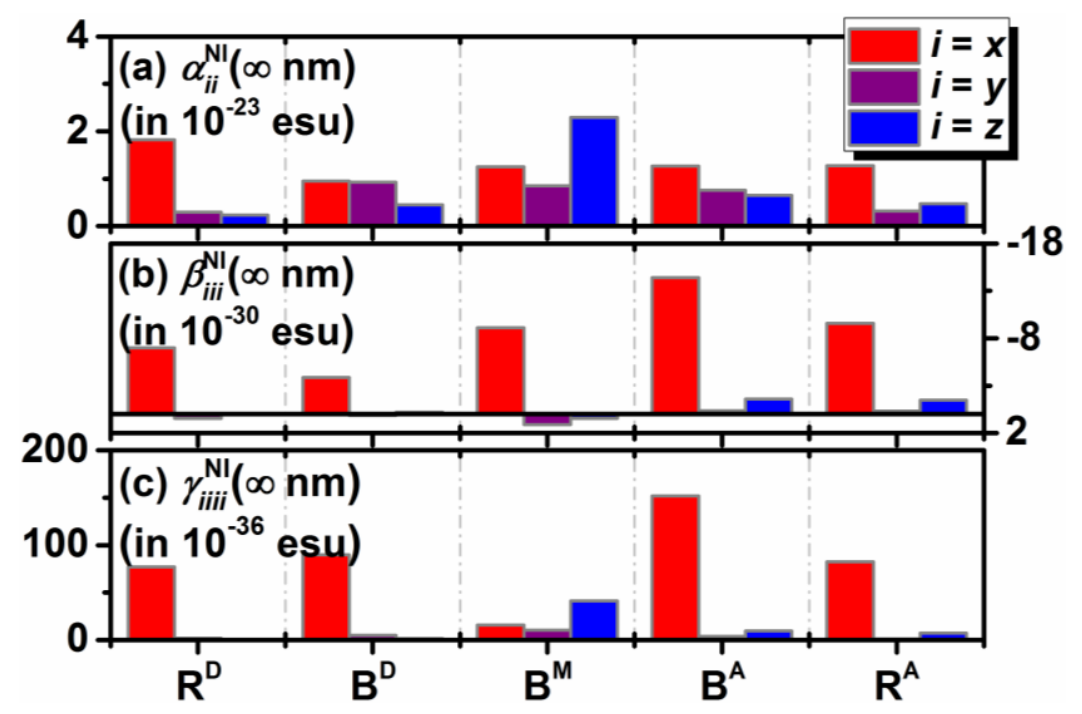

Figure 5. Decomposition of (a) the polarizability components, (b) the first-order hyperpolarizability components, and (c) the second-order hyperpolarizability components into each constituent unit by integrating the local contributions of the (hyper)polarizability tensor. Capital letters on the horizontal axis represent the units that make up the MIC, as designated in Scheme 1.

(Hyper)polarizability comparable with EFISHG-experiments The isotropic average polarizability $\left[\alpha_{\text {iso }}(\lambda)\right.$ ], projection of first-order hyperpolarizability on dipole moment $\left[\beta_{\text {vec }}(\lambda)\right]$, and scalar component of second-order hyperpolarizability $\left[\gamma_{\|}(\lambda)\right]$ are the most practical quantities and can be directly compared with EFISHG values. The three quantities of the studied MIC are listed in Table S4 and illustrated in Figure 6. 
For a particular solvent in Figure 6(a), the $\alpha_{\text {iso }}(\infty)$ under different external fields is almost the same, that is to say, there is no obvious resonance of the molecular polarizability under the applied electric field. Generally speaking, the polarizability of molecules is indeed insensitive to the external field, which has been observed in our previous studies on optical nonlinearity of other systems. ${ }^{6,54}$ Under the same electric field, on the other side, the $\alpha_{\text {iso }}(\infty)$ of the molecule calculated in solvent is slightly higher than that in vacuum, but there is little difference of it in different sorts of solvents.
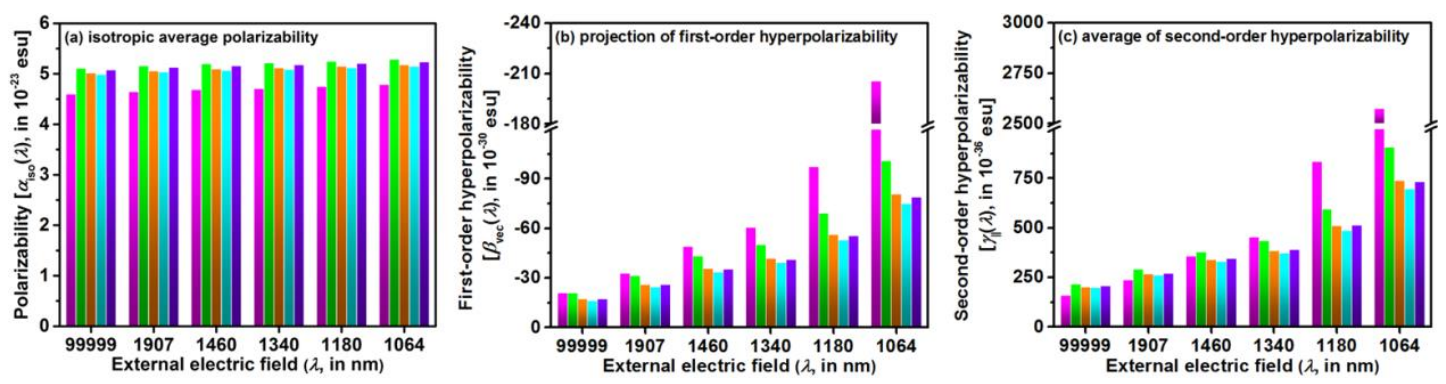

Figure 6. Diagrams of the (hyper)polarizability comparable with EFISHG-xperiments: (a) polarizabilities $\left[\alpha_{\text {iso }}(\lambda)\right]$, (b) first-order hyperpolarizabilities $\left[\beta_{\text {vec }}(\lambda)\right]$, and (c) second-order hyperpolarizabilities $\left[\gamma_{\|}(\lambda)\right]$ of the MIC. The legend is consistent with that in Figure 2(a).

By comparing Figure 6(b) and (c), it can be seen that the external field resonance or solvation effect has the similar effect on $\beta_{\text {vec }}(\lambda)$ and $\gamma_{\|}(\lambda)$, which trend similar to that of the $\beta_{\mathrm{HRS}}(\lambda)$ in response to these influence discussed above. To be exact, the hyperpolarizability of the MIC in various environments increases with the increase of frequency in external field. However, only under the action of higher-frequency external field, the difference of hyperpolarizability in different solvents becomes significant. The molecular hyperpolarizability in vacuum is most sensitive to frequency of incident light, and it changes from indistinguishable with that in solvents at zero-frequency limit to absolute superiority at $1064 \mathrm{~nm}$ fundamental wavelength. This trend can be well explained by the conclusion deduced from the two-state model 55 that the dynamic perturbation to the hyperpolarizability of chromophores is expected to reach the maximum by excitation using photons with twice the 
wavelength of one-photon transition, because the incident wavelength at $1064 \mathrm{~nm}$ is exactly twice the maximum absorption wavelength of the studied MIC in vacuum.

The (hyper)polarizability tensors in Figure 7 display maximum magnitudes in the $x-z$ plane, which is consistent with the (hyper)polarizability component and (hyper)polarizability density analyses above. Especially in the four corners of Figure 7(b) and (c), the direction of tensor vectors is obviously not perpendicular to the sphere, which indicates that the change direction of dipole moment in these regions is different from that of applied field. The above facts clearly show the anisotropy of the (hyper)polarizability of the system.

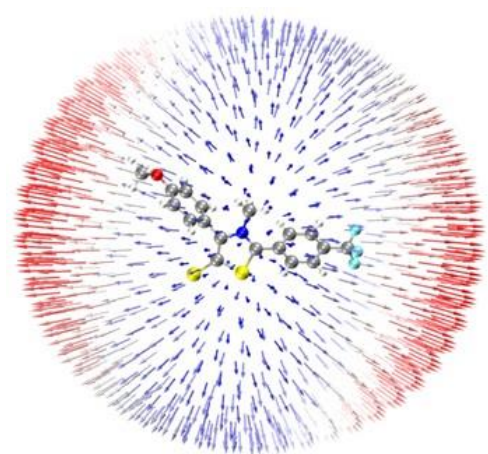

(a)

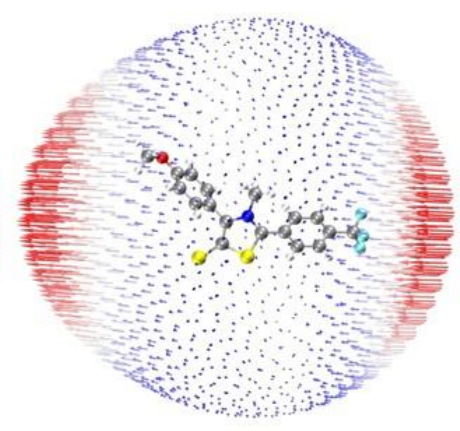

(b)

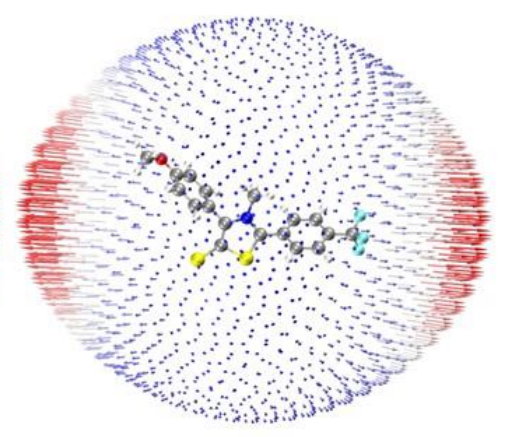

(c)

Figure 7. Unit sphere representation of (a) the polarizability tensor, (b) the first-order hyperpolarizability tensor, and (c) the second-order hyperpolarizability tensor of the MIC. The length and the colour of the arrows represent magnitude of (hyper)polarizability in different directions.

\section{Conclusion}

The effects of external field wavelength and solvation on the optical properties of a mesoionic compound, MIC, with push-pull electronic structure were studied in detail by means of quantum chemistry calculations. The solvation effect leads to a significant blue-shift of the electron absorption spectra of the MIC in the solution compared with that in vacuum, but no obvious difference is observed in the spectra in different solvents. The NTO analysis identified the contribution to the maximum absorption of the MIC originating from the $n \rightarrow \pi^{*}$ and $\pi \rightarrow \pi^{*}$ transitions at the mesoionic ring and benzene unit adjacent to the electron acceptor, and the conclusion from frontier molecular orbital analysis and hole-electron analysis are very consistent with it. The HRS-derived first-order hyperpolarizability and the polarization scan of 
the HRS intensity enlarge with the increase of the external field frequency but reduce as the polarity of the environment increases, which reveals the induced strengthening and weakening effects on the response properties of electric field and solvation to the studied system, respectively. In the coordinate system of the molecule, the (hyper)polarizability component on the $x$-axis makes a great contribution to EFISHGderived response properties. In contrast, the contribution of the $z$-axis component can not be ignored, while $y$-component is almost zero. Detailed (hyper)polarizability density and (hyper)polarizability decomposition analysis reveal the contribution of each structural unit of the studied molecule to its (hyper)polarizability component in terms of visualization and quantification analyses, respectively. In addition, the molecular (hyper)polarizabilities which can be compared with the EFISHG experiments were predicted, and it is found that their response to the environment is exactly the same as the trend of HRS-derived hyperpolarizability discussed. This work provides a physical perspective for understanding the photophysical property and optical nonlinearity for mesoionic compounds in different external field and solvent environments, which will be helpful to design high-performance optoelectronic materials with stimulus response.

\section{Supporting Information Available:}

Detailed formulas for calculating molecular response properties; optimized Cartesian coordinates for ground-state MIC; selected data related to electron transitions for the MIC; HOMOs and LUMOs for MIC; selected parameters related to the HRS study of the MIC; harmonic light intensity $\left(\mathrm{I}_{\Psi \mathrm{V}}^{2 \omega}\right)$ for the MIC as a function of the polarization angle $(\Psi)$ by polar representation; selected parameters related to the EFISHG technique of the studied MIC; plots of (hyper)polarizability density functions of the MIC; components of the (hyper)polarizability of the constituent units of the MIC.

\section{Acknowledgments}

This work was supported by The Natural Science Foundation of the Jiangsu Higher Education Institutions of China (Grant No. 18KJA180005). 


\section{References}

(1) Ostroverkhova, O. Organic Optoelectronic Materials: Mechanisms and Applications. Chem. Rev. 2016, 116, 13279-13412.

(2) Dong, H.; Zhu, H.; Meng, Q.; Gong, X.; Hu, W. Organic Photoresponse Materials and Devices. Chem. Soc. Rev. 2012, 41, 1754-1808.

(3) Mutailipu, M.; Poeppelmeier, K. R.; Pan, S. Borates: A Rich Source for Optical Materials. Chem. Rev. 2020, DOI: 10.1021/acs.chemrev.0c00796.

(4) Zardo, I.; Yazji, S.; Marini, C.; Uccelli, E.; Morral, A. F. i; Abstreiter, G.; Postorino, P. Pressure Tuning of the Optical Properties of GaAs Nanowires. ACS Nano 2012, 6, 3284-3291.

(5) Reczyński, M.; Nakabayashi, K.; Ohkoshi, S.-i. Tuning the Optical Properties of Magnetic Materials. Eur. J. Inorg. Chem. 2020, 2669-2678.

(6) Liu, Z.; Lu, T. Controllable Photophysical and Nonlinear Properties in Conformation Isomerization of Macrocyclic [32] Octaphyrin(1.0.1.0.1.0.1.0) Involving Hückel and Möbius Topologies. J. Phys. Chem. C 2020, 124, 845-853.

(7) Biswas, T.; Ravindra, P.; Athresh, E.; Ranjan, R.; Avasthi, S.; Jain, M. Optical Properties of $\mathrm{Zn}_{2} \mathrm{Mo}_{3} \mathrm{O}_{8}$ : Combination of Theoretical and Experimental Study. J. Phys. Chem. C 2017, 121, 24766-24773.

(8) Gong, P.; Luo, S.; Yang, Y.; Liang, F.; Zhang, S.; Zhao, S.; Luo, J.; Lin, Z. Nonlinear Optical Crystal $\mathrm{Rb}_{4} \mathrm{Sn}_{3} \mathrm{Cl}_{2} \mathrm{Br}_{8}$ : Synthesis, Structure, and Characterization. Cryst. Growth Des. 2018, 18, 380-385.

(9) Mutailipu, M.; Zhang, M.; Zhang, B.; Wang, L.; Yang, Z.; Zhou, X.; Pan, S. $\mathrm{SrB}_{5} \mathrm{O}_{7} \mathrm{~F}_{3}$ Functionalized with $\left[\mathrm{B}_{5} \mathrm{O}_{9} \mathrm{~F}_{3}\right]^{6-}$ Chromophores: Accelerating the Rational Design of Deep-Ultraviolet Nonlinear Optical Materials. Angew. Chem., Int. Ed. 2018, $57,6095-6099$.

(10) Filatov, M. A.; Baluschev, S.; Ilieva, I. Z.; Enkelmann, V.; Miteva, T.; Landfester, K.; Aleshchenkov, S. E.; Cheprakov, A. V. Tetraaryltetraanthra[2,3]porphyrins: Synthesis, Structure, and Optical Properties. $J$. Org. Chem. 2012, 77, 11119-11131.

(11) Liu, Z.; Hua, S.; Yan, X. Linear and Nonlinear Optical Properties of Triphenylamine-Indandione Chromophores: Theoretical Study of the StructureFunction Relationship under the Combined Action of Substituent and Symmetry Change. J. Phys. Chem. A 2018, 122, 2344-2352. 
(12) Vesga, Y.; Diaz, C.; Crassous, J.; Hernandez, F. E. Two-Photon Absorption and Two-Photon Circular Dichroism of a Hexahelicene Derivative with a Terminal Donor-Phenyl-Acceptor Motif. J. Phys. Chem. A 2018, 122, 3365-3373.

(13) Liu, Z.; Hua, S.; Wu, G. Extended First Hyperpolarizability of Quasi-Octupolar Molecules by Halogenated Methylation: Whether the Iodine Atom is the Best Choice. J. Phys. Chem. C 2018, 122, 21548-21556.

(14) Knoppe, S.; Verbiest, T. Resonance Enhancement of Nonlinear Optical Scattering in Monolayer-Protected Gold Clusters. J. Am. Chem. Soc. 2017, 139, $14853-14856$.

(15) Ferrari, P.; Upadhyay, S.; Shestakov, M. V.; Vanbuel, J.; De Roo, B.; Kuang, Y.;

Di Vece, M.; Moshchalkov, V. V.; Locquet, J.-P.; Lievens, P.; Janssens, E. Wavelength-Dependent Nonlinear Optical Properties of Ag Nanoparticles Dispersed in a Glass Host. J. Phys. Chem. C 2017, 121, 27580-27589.

(16) Dye, J. L. Electrons as Anions. Science 2003, 301, 607-608.

(17) Liu, Z.; Yan, X.; Li, L.; Wu, G. Theoretical Investigation of the Topology and Metalation Effects on the First Hyperpolarizability of Rosarins. Chem. Phys. Lett. 2015, 641, 5-8.

(18) Liu, Z.; Lu, T. Optical Properties of Novel Conjugated Nanohoops: Revealing the Effects of Topology and Size. J. Phys. Chem. C 2020, 124, 7353-7360.

(19) Liu, Z.; Lu, T.; Chen, Q. An sp-Hybridized All-Carboatomic Ring, Cyclo[18]carbon: Electronic Structure, Electronic Spectrum, and Optical Nonlinearity. Carbon 2020, 165, 461-467.

(20) Niu, X.; Gautam, P.; Kuang, Z.; Yu, C. P.; Guo, Y.; Song, H.; Guo, Q.; Chan, J. M. W.; Xia, A. Intramolecular Charge Transfer and Solvation Dynamics of Push-Pull Dyes with Different $\pi$-Conjugated Linkers. Phys. Chem. Chem. Phys. 2019, 21, $17323-17331$.

(21) Liu, Z.; Yan, X.; Li, L.; Wu, G. Modulation of the Optical Properties of D- $\pi-A$ Type Azobenzene Derivatives by Changing the $\pi$-Conjugated Backbone: A Theoretical Study. J. Theor. Comput. Chem. 2015, 14, 1550041.

(22) Sutradhar, T.; Misra, A. The Role of $\pi$-Linkers and Electron Acceptors in Tuning the Nonlinear Optical Properties of BODIPY-Based Zwitterionic Molecules. RSC Adv. 2020, 10, 40300-40309. 
(23) Liu, Z.; Yan, X.; Li, L.; Wu, G. Theoretical Insight into the Substituent Effects on Linear and Nonlinear Optical Properties of Azobenzene-Based Chromophores. $J$. Phys. Org. Chem., 2017, 30, e3631.

(24) Lescos, L.; Sitkiewicz, S. P.; Beaujean, P.; Blanchard-Desce, M.; Champagne, B.; Matito, E.; Castet, F. Performance of DFT Functionals for Calculating the SecondOrder Nonlinear Optical Properties of Dipolar Merocyanines. Phys. Chem. Chem. Phys. 2020, 22, 16579-16594.

(25) Baker, W.; Ollis, W. D.; Poole, V. D. Cyclic Meso-Ionic Compounds. Part I. The Structure of the Sydnones and Related Compounds. J. Chem. Soc. 1949, 307-314.

(26) Baker, W.; Ollis, W. D.; Poole, V. D. Cyclic Meso-Ionic Compounds. Part III. Further Properties of the Sydnones and the Mechanism of Their Formation. J. Chem. Soc. 1950, 1542-1551.

(27) Moura, G. L.; Simas, A. M.; Miller, J. Mesoionic Rings as Efficient Asymmetric Bridges for the Design of Compounds with Large Optical Nonlinearities. Chem. Phys. Lett. 1996, 257, 639-646.

(28) Silva, A. M. S.; da Rocha, G. B.; Menezes, P. H.; Miller, J.; Simas, A. M. Theoretical Nonlinear Optics Equivalence Between Mesoionic and Polyenic Bridges in Push-Pull Compounds. J. Braz. Chem. Soc. 2005, 16, 583-588.

(29) de Oliveira, M. B.; Miller, J.; Pereira, A. B.; Galembeck, S. E.; de Moura, G. L.; Simas, A. M. Mesoionic 2-N-Cycloalkylamino-5-Alkyl-1, 3-Dithiolium-4-Thiolates. Phosphorus Sulfur Silicon Relat. Elem. 1996, 108, 75-84.

(30) Rakov, N.; de Araújo, C. B.; da Rocha, G. B.; Simas, A. M.; Athayde-Filho, P. A. F.; Miller, J. Two-Photon Absorption in Mesoionic Compounds Pumped at the Visible and at the Infrared. Chem. Phys. Lett. 2000, 332, 13-18.

(31) Rakov, N.; de Araújo, C. B.; Rocha, G. B.; Simas, A. M.; Athayde-Filho, P. A.; Miller, J. Reverse Saturable Absorption and Anti-Stokes Fluorescence in Mesoionic Compounds Pumped at 532 nm. Appl. Opt. 2001, 40, 1389-1395.

(32) Barbosa-Silva, R.; Nogueira, M. A. M.; Souza, H. D. S.; Lira, B. F; de AthaydeFilho, P. F.; de Araújo, C. B. First Hyperpolarizability of 1,3-Thiazolium-5-Thiolates Mesoionic Compounds. J. Phys. Chem. C 2019, 123, 677-683.

(33) Liu, Z.; Tian, Z.; Lu, T.; Hua, S. Theoretical Framework of 1,3-Thiazolium-5Thiolates Mesoionic Compounds: Exploring the Nature of Photophysical Property and Molecular Nonlinearity. J. Phys. Chem. A 2020, 124, 5563-5569. 
(34) Lyra, B. F.; de Morais, S. A.; Rocha, G. B.; Miller, J.; Moura, G. L. C.; Simas, A. M.; Peppe, C.; de Athayde-Filho, P. F. 1, 3-Thiazolium-5-Thiolates Mesoionic Compounds: Semiempirical Evaluation of Their First Static Hyperpolarizabilities and Synthesis of New Examples. J. Braz. Chem. Soc. 2010, 21, 934-940.

(35) Perdew, J. P.; Burke, K.; Ernzerhof, M. Generalized Gradient Approximation Made Simple. Phys. Rev. Lett. 1996, 77, 3865-3868.

(36) Schäfer, A.; Huber, C.; Ahlrichs, R. Fully Optimized Contracted Gaussian Basis Sets of Triple Zeta Valence Quality for Atoms Li to Kr. J. Chem. Phys. 1994, 100, $5829-5835$.

(37) Marenich, A. V.; Cramer, C. J.; Truhlar, D. G. Universal Solvation Model based on Solute Electron Density and a Continuum Model of the Solvent Defined by the Bulk Dielectric Constant and Atomic Surface Tensions. J. Phys. Chem. B 2009, 113, $6378-6396$.

(38) Casida, M. K.; Jamorski, C.; Casida, K. C.; Salahub, D. R. Molecular Excitation Energies to High-Lying Bound States from Time-Dependent Density-Functional Response Theory: Characterization and Correction of the Time-Dependent Local Density Approximation Ionization Threshold. J. Chem. Phys. 1998, 108, 4439-4449.

(39) Stratmann, R. E.; Scuseria, G. E.; Frisch, M. J. An Efficient Implementation of Time-Dependent Density-Functional Theory for the Calculation of Excitation Energies of Large Molecules. J. Chem. Phys. 1998, 109, 8218-8224.

(40) Weigend, F.; Ahlrichs, R. Balanced Basis Set of Split Valence, Triple Zeta and Quadruple Zeta Valence Quality for H to Rn: Design and Assessment of Accuracy, Phys. Chem. Chem. Phys. 2005, 7, 3297-3305.

(41) Jensen F. Introduction to computational chemistry. John Wiley \& Sons, West Sussex, 2017.

(42) Yanai, T.; Tew, D.; Handy, N. A New Hybrid Exchange-Correlation Functional using the Coulomb-Attenuating Method (CAM-B3LYP). Chem. Phys. Lett. 2004, 393, $51-57$.

(43) Dunning Jr., T. H. Gaussian Basis Sets for Use in Correlated Molecular Calculations. I. The Atoms Boron through Neon and Hydrogen. J. Chem. Phys. 1989, 90, 1007-1023.

(44) Torrent-Sucarrat, M.; Anglada, J. M.; Luis, J. M. Evaluation of the Nonlinear Optical Properties for An Expanded Porphyrin Hückel-Möbius Aromaticity Switch. $J$. Chem. Phys. 2012, 137, 184306. 
(45) Nénon, S.; Champagne, B.; Spassova, M. I. Assessing Long-Range Corrected Functional with Physically-Adjusted Range-Separated Parameters for Calculating the Polarizability and the Second Hyperpolarizability of Polydiacetylene and Polybutatriene Chains. Phys. Chem. Chem. Phys. 2014, 16, 7083-7088.

(46) Torrent-Sucarrat, M.; Anglada, J. M.; Luis, J. M. Evaluation of the Nonlinear Optical Properties for Annulenes with Hückel and Möbius Topologies. J. Chem. Theory Comput. 2011, 7, 3935-3943.

(47) Becke, A. D. A Multicenter Numerical Integration Scheme for Polyatomic Molecules. J. Chem. Phys.1988, 88, 2547-2553.

(48) Frisch, M. J.; Trucks, G. W.; Schlegel, H. B.; Scuseria, G. E.; Robb, M. A.; Cheeseman, J. R.; Scalmani, G.; Barone, V.; Petersson, G. A.; Nakatsuji, H.; et al. Gaussian 16, revision A.03; Gaussian, Inc.: Wallingford, CT, 2016.

(49) Lu, T.; Chen, F. Multiwfn: A Multifunctional Wavefunction Analyzer. J. Comput. Chem. 2012, 33, 580-592.

(50) Lu, T. Multiwfn, version 3.7(dev). http://sobereva.com/multiwfn/ (accessed Sep. 9, 2019).

(51) Humphrey, W.; Dalke, A.; Schulten, K. VMD: Visual Molecular Dynamics. J. Mol. Graphics 1996, 14, 33-38.

(52) Martin, R. L. Natural Transition Orbitals. J. Chem. Phys. 2003, 118, 4775-4777. (53) Lu T. Multiwfn manual, version 3.7, Section 3.21.1, available at http://sobereva.com/multiwfn/ (accessed on Dec 1, 2020).

(54) Liu, Z.; Hua, S.; Wu, G. Extended First Hyperpolarizability of Quasi-Octupolar Molecules by Halogenated Methylation: Whether the Iodine Atom is the Best Choice, J. Phys. Chem. C 2018, 122, 21548-21556.

(55) Bersohn, R.; Pao, Y. H.; Frisch, H. L. Double-Quantum Light Scattering by Molecules. J. Chem. Phys. 1966, 45, 3184-3198. 